\title{
Field test and preliminary analysis of a combined diurnal solar heating and nocturnal radiative cooling system
}

\author{
Mingke $\mathrm{Hu}^{\text {a }}$, Gang Pei *, a, Qiliang Wang a , Jing $\mathrm{Li}^{\text {a }}$, Yunyun Wang ${ }^{\text {a, b }}$, Jie $\mathrm{Ji}^{\text {a }}$ \\ a Department of Thermal Science and Energy Engineering, University of Science and Technology of China, Hefei 230027, China \\ ${ }^{\mathrm{b}}$ Key Laboratory of Optoelectronic Devices and Systems of Ministry of Education and Guangdong Province, College of \\ Optoelectronic Engineering, Shenzhen University, Shenzhen 518060, China
}

* Corresponding author. Tel.: 0551-63601652. E-mail address: peigang@ustc.edu.cn

\begin{abstract}
A type of composite surface was manufactured for trial to achieve integrated solar heating and radiative cooling functions. The spectral properties of the composite surface present a relatively clear selectivity in the spectra of solar heating and radiation cooling wavelengths. A combined system for both solar heating and radiative cooling (named SH-RC system) based on the composite surface was mounted together with a traditional flat-plate solar heating system. Comparative experiments were carried out to investigate their thermal performances both at daytime and nighttime. Results showed that the composite surface has a relatively evident spectral selectivity. In diurnal collector testing mode, the thermal efficiency of the SH-RC collector was $62.7 \%$ at zero-reduced temperature, which was about $86.4 \%$ of that of the traditional flat-plate solar heating collector. In nocturnal collector testing mode, the SH-RC collector had net radiative cooling powers of $50.3 \mathrm{~W} / \mathrm{m}^{2}$ on a clear night and 23.4 $\mathrm{W} / \mathrm{m}^{2}$ on an overcast night; by contrast, the traditional flat-plate solar heating collector exhibited very little radiative cooling capacity. In diurnal system testing mode, the daily average thermal efficiency of
\end{abstract}


the SH-RC system and the traditional flat-plate solar heating system at zero-reduced temperature was $38.6 \%$ and $48.4 \%$, respectively. Based on experimental results, the SH-RC system showed a considerable performance for both diurnal solar heating and nocturnal radiative cooling.

KEYWORDS: Solar heating; Radiative cooling; Atmospheric window; Thermal performance.

\section{Introduction}

With the excessive exploitation of natural fossil resources like coal, oil, and natural gas, along with heavy pollution, environmental and energy-related problems have become the world's major concerns [1]. Solar energy is a renewable and green energy considered to be a promising substitute for increasingly exhausted traditional energy resources ${ }^{[2]}$. Naturally, an increasing number of researchers focus on solar energy utilization ${ }^{[3-8]}$. Photovoltaic and photo-thermal technologies are the main applications of solar energy that are developed rapidly in recent years ${ }^{[9-13]}$. Photo-thermal technologies are the most developed solar applications today ${ }^{[14-16]}$. The largest proportion of energy consumed by buildings come from space heating and cooling, which now occupies a significant share of society's total energy consumption ${ }^{[17]}$. Meanwhile, a large area is required for the utilization of solar energy because of its intermittency and low energy intensity. Solar energy installations can be integrated with building envelopes easily and can significantly reduce building energy consumption; thus, integrating solar energy with buildings is a necessity ${ }^{[18,19]}$.

In the majority of wave bands, the atmosphere weakens heat radiation from the ground to the cold outer space because of its low transmittance. However, in bands such as $0.3-3,3.2-4.8$, and $8-13 \mu \mathrm{m}$, the atmosphere has extremely high transmittance ${ }^{[20]}$. The wavelength of interest is $8-13 \mu \mathrm{m}$ (i.e., the atmospheric window), where the heat radiation of bodies on the ground is mainly concentrated ${ }^{[21]}$. The phenomenon that objects on the surface of the earth obtain a cooling power by radiating heat to outer 
space through the atmospheric window is called spectrally selective radiative cooling (short for "radiative cooling" in the followings) ${ }^{[22]}$. Radiative cooling technology has also attracted the attention of a growing number of researchers in recent years because of its advantages in energy conservation and emission reduction ${ }^{[20-34]}$. Radiative cooling systems are simple, making them easily well-integrated with building envelopes. Moreover, radiative cooling is a totally passive cooling technology and thus can obtain cooling powers without any external driving energy inputs. However, their cooling powers are not sufficiently large. Basically, a radiative cooling device cannot act as a solar collector during daytime because the radiator has extremely low spectral absorptivity in the solar radiation wavelength (i.e., $0.2-3 \mu \mathrm{m}$ ) ${ }^{[32]}$. Conversely and interestingly, a traditional solar collector cannot act as a nocturnal cooling radiator because of its extremely low emissivity in the atmospheric window wavelength.

Inspired by this, it is significant and challenging to offer a system that can act not only as a solar heating system in the daytime but also a as a radiative cooling system in the nighttime. This system could work all day to address the limitations of traditional radiative cooling devices and flat plate solar collectors mentioned above and shorten the initial payback period. To carry out solar heating at daytime and radiative cooling at nighttime on the same system, a spectrally selective surface that can achieve the two functions should be obtained. This specific surface should have high spectral absorptivity (emissivity) for solar radiation and atmospheric window wavelengths so that it could obtain a large heating power during daytime and a large cooling power at nighttime. In addition, the surface should have low spectral absorptivity (emissivity) in other bands aside from solar radiation and atmospheric window wavelengths, allowing it to reduce daily radiant heating loss and nightly radiant cooling loss by exchanging heat with the sky and the surroundings. The ideal surface for both solar 
heating and radiative cooling should have absorptivity (emissivity) of $100 \%$ in solar radiation and atmospheric window wavelengths and zero in other bands, as shown in Fig. 1.

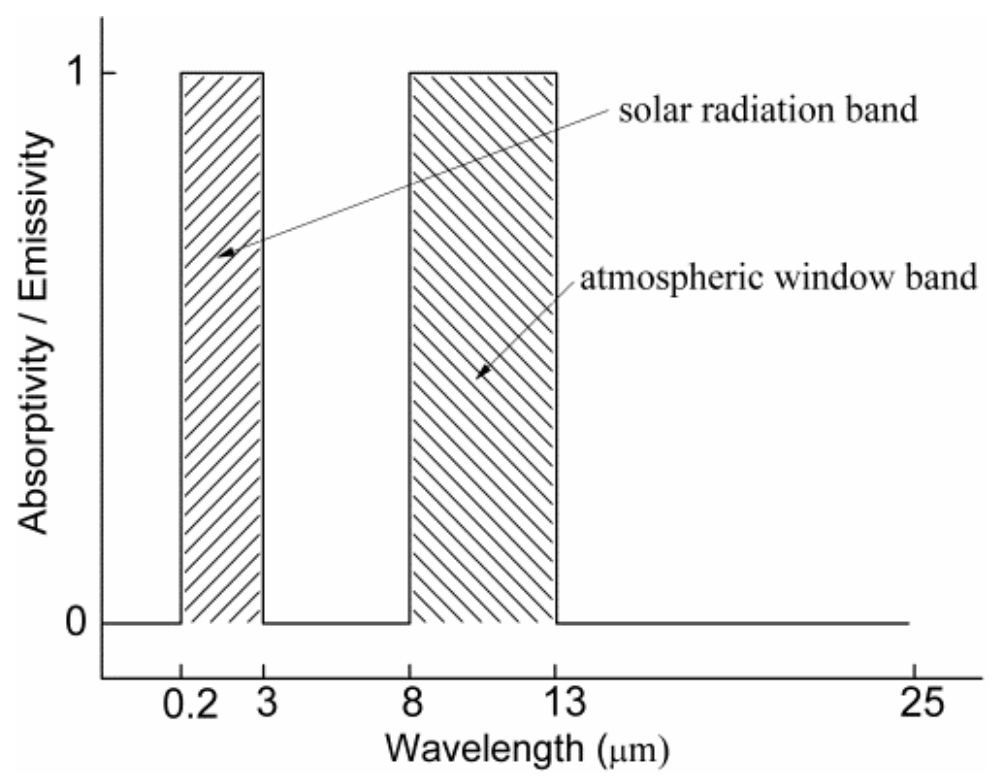

Fig. 1 Spectral absorptivity (emissivity) of the ideal surface for both solar heating and radiative cooling

To the best of our knowledge, reports on diurnal solar heating and nocturnal radiative cooling using the same system are few. An original study by Matsuta et al. ${ }^{[33]}$ used a type of collective-radiative surface made of a black copper plate, which was coated with a thin polyvinyl-difluoride film that acted as a solar collector at daytime and a sky radiator at night. They continuously investigated its heating and cooling performance together with a non-selective solar collector-sky radiator for $22 \mathrm{~h}$, from 9:00 a.m. to 7:00 a.m. of the next day. However, their study lacks long-term experiments and radiative cooling tests under different sky conditions. In this study, we proposed a new spectrally selective surface for both solar heating during daytime and radiative cooling during nighttime. We trial-manufactured a type of composite surface named TPET composite surface by combining Ti-based solar selective absorber with polyethylene terephthalate (PET) based on the spectral analysis mentioned above. A schematic diagram of the TPET composite surface is shown in Fig. 2. The Ti-based solar absorber (Fig. 3a) employed in this study (a product bought from Guangdong Five Star Solar Energy Co. Ltd) consists of a $400-\mu$ m-thick aluminum substrate and a 200 -nm-thick 
Ti-based absorbing coating. The Ti-based absorbing coating is an evaporated titanium nitride film $\left(\mathrm{TiNO}_{\mathrm{x}}\right)$ synthesized by the vacuum magnetron sputtering technology. At the beginning, we cleaned the surface of the Ti-based solar absorber and made it as flat as possible. Then we covered a layer of 30- $\mu$ m-thick PET powder (Fig. 3b) on the Ti-based solar absorbing coating with a powder monitor. At the next stage, it was sent to a constant temperature oven (Fig. 3c) at a temperature of $200{ }^{\circ} \mathrm{C}$ for approximately $10 \mathrm{~min}$. The PET powder was turned into film and then adhered to the Ti-based solar absorber. The new composite surface after natural cooling was called the TPET composite surface (Fig. 4).

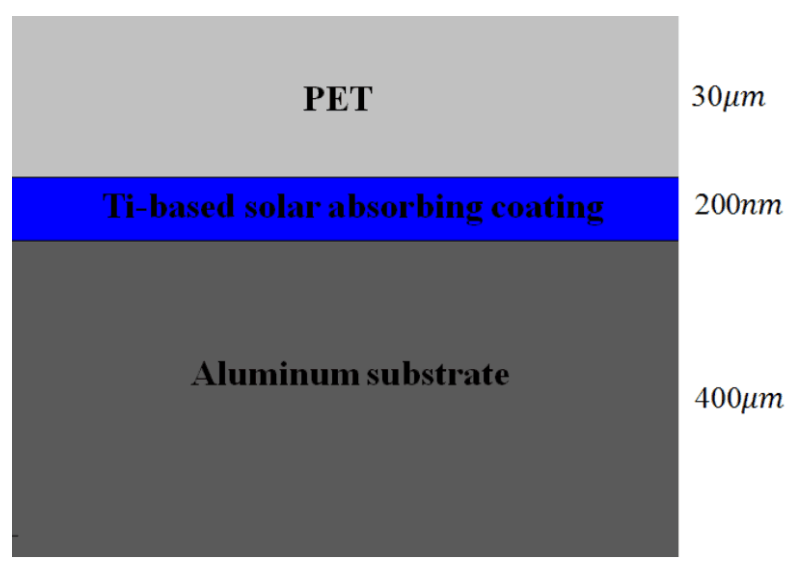

Fig. 2. Schematic diagram of the TPET composite surface

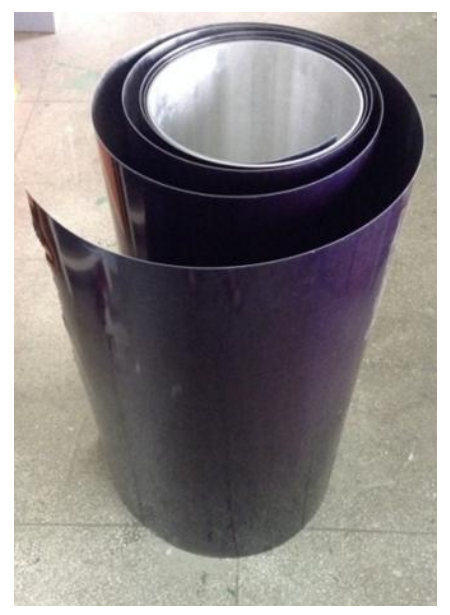

(a) Ti-based solar absorber

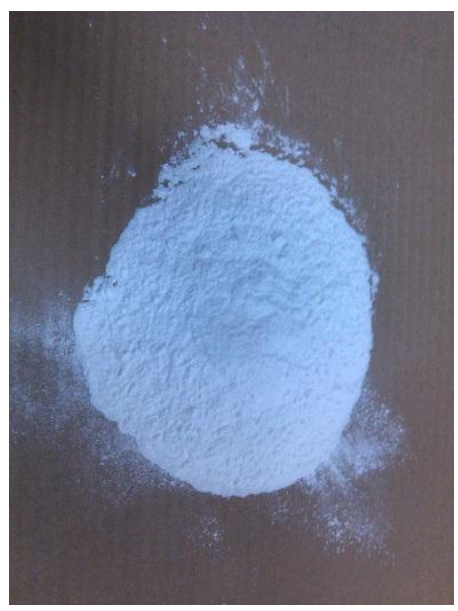

(b) PET powder

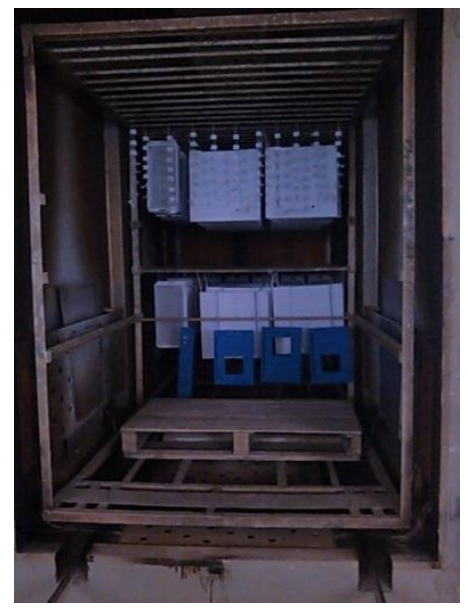

(c) Constant temperature oven

Fig. 3. Materials and processing facility of the TPET composite surface 


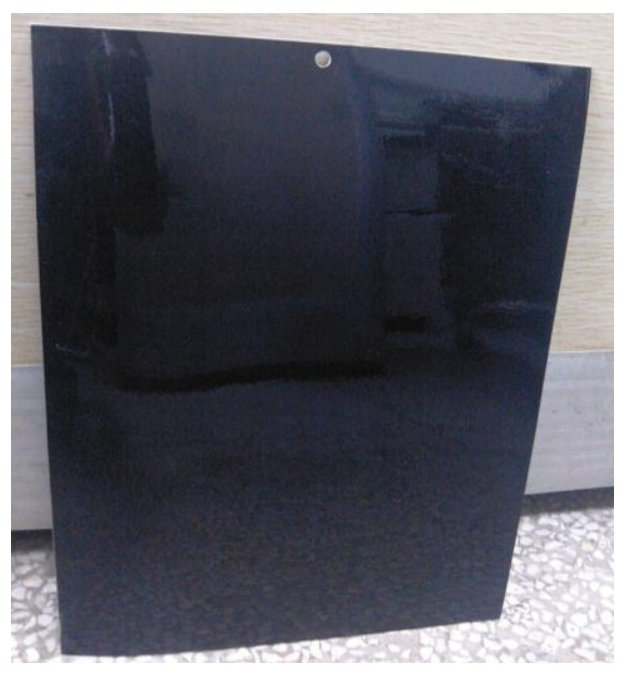

Fig.4. The photo of the TPET composite surface.

Then, the spectral properties of the TPET composite surface were measured using spectral testing instruments. In addition, a combined system for both solar heating and radiative cooling (SH-RC system) based on the TPET composite surface was mounted beside a traditional flat-plate solar heating system. Long-term comparative experiments were carried out under different sky conditions to fully investigate their thermal performances at daytime and nighttime.

\section{Description of the solar heating and radiative cooling collector (SH-RC collector) and the}

\section{experimental set-up}

\subsection{Description of the SH-RC collector}

We designed two corresponding collectors, namely, a SH-RC collector (collector A) and a traditional flat-plate solar collector (collector B), to evaluate the thermal performance between the SH-RC system (system A) and traditional flat-plate solar heating system (system B). In Fig. 5 it shows the section structure of the two collectors with the same components, such as windscreen, collecting surface, copper pipes, and insulation layer. The collecting surface has an area of $1140 \mathrm{~mm} \times 950 \mathrm{~mm}$ and a thickness of about $0.4 \mathrm{~mm}$. Nine copper pipes, with dimensions of $\phi 7 \mathrm{~mm} \times 1 \mathrm{~mm} \times 1220 \mathrm{~mm}$ were placed at the back of the collecting surface evenly. The length between the two adjacent copper 
pipes was approximately $105.6 \mathrm{~mm}$. Anterior to the collecting surface, a windscreen was provided to prevent heating or cooling loss by heat convection and to prevent the entry of dust particles and rain. The air gap between the windscreen and collecting surface was $40 \mathrm{~mm}$. Additionally, a $50 \mathrm{~mm}$ thick thermal insulation layer was placed behind and around the collecting surface.

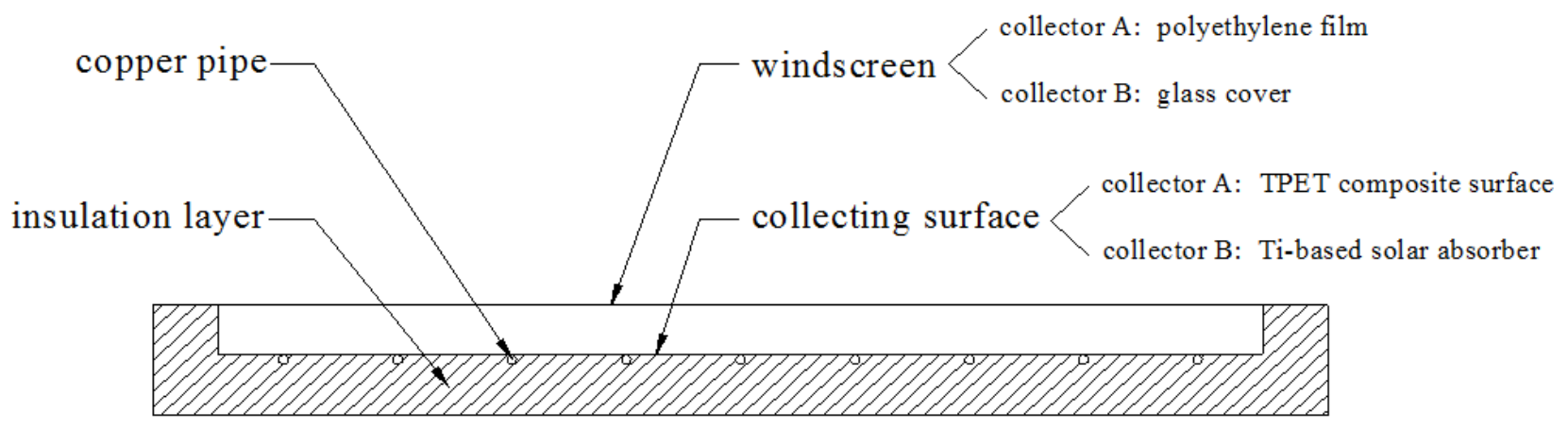

Fig. 5. Section structure of the two collectors

However, the two collectors have different materials for the collecting surface and windscreen; the collecting surface of collector A is the TPET composite surface, whereas that of collector B is the Ti-based solar selective absorber. We tested the spectral properties of the TPET composite surface and Ti-based solar absorber first. The spectral testing instruments used in this study were the UV-Vis-NIR spectrophotometer (DUV-3700) (Fig. 6a) and Fourier transform infrared spectrophotometer (FTIR) (Bruker Equinox 55) (Fig. 6b). Both instruments can test spectral reflectivity and transmittance as a function of wavelength $\lambda$, with spectral regions at $0.2-2.5$ and $2.5-25 \mu \mathrm{m}$, respectively. We tested the samples of the two surfaces three times under the same conditions and took the average to remove any discrepancies. Fig. 7 shows their spectral absorptivities (emissivities) based on the testing results and Equation (1).

$$
\alpha_{\lambda}+\rho_{\lambda}=1
$$




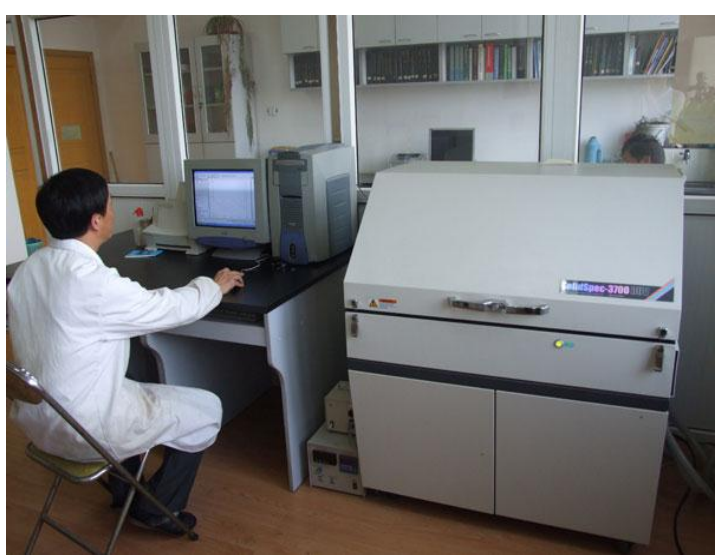

(a) UV-Vis-NIR spectrophotometer (DUV-3700)

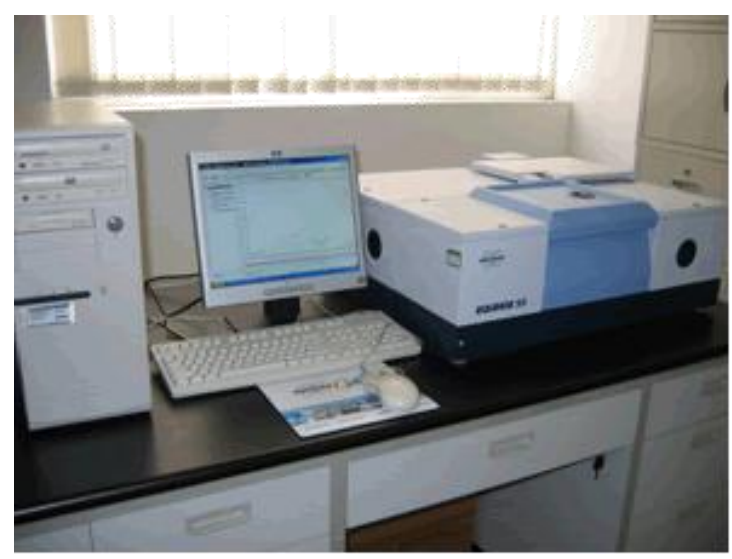

(b) Fourier transform infrared spectrophotometer (Bruker Equinox 55)

Fig.6. The spectral testing instruments

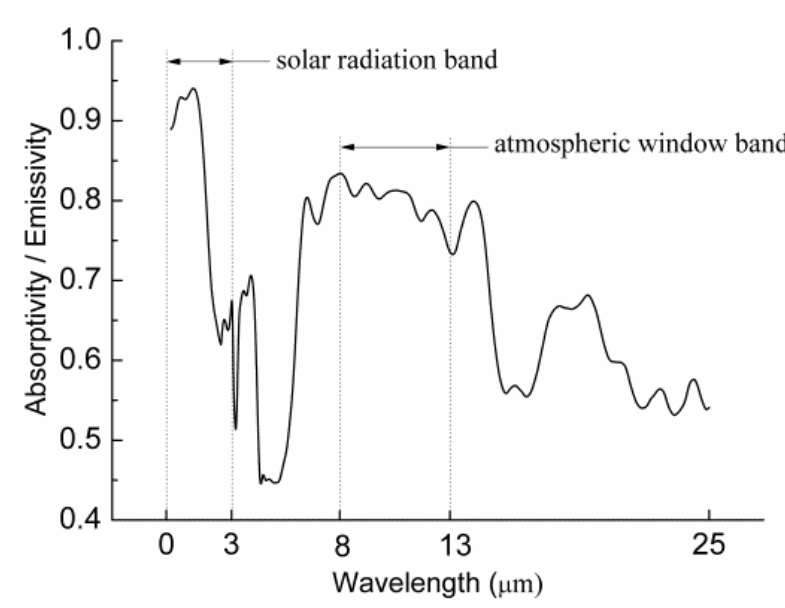

(a) TPET composite surface (in collector A)

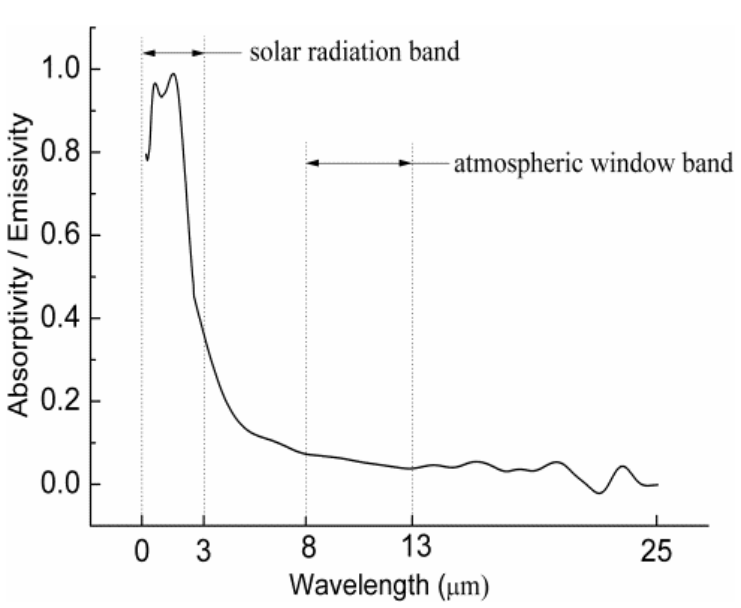

(b) Ti-based solar absorber (in collector B)

Fig. 7. Spectral absorptivity (emissivity) of the two collecting surfaces

A glass plate acts as the windscreen of collector $\mathrm{B}$, which has a spectral transmittance of over $80 \%$ in the solar radiation band and a very low spectral transmittance in the infrared band. Notably, the windscreen of collector A should have high spectral transmittance in both solar radiation and atmospheric window wavelengths to enable most radiation in the two regions to pass through it. Therefore, the glass cover used in collector B cannot act as a windscreen here. Polyethylene film has a high spectral transmittance in the entire $0.2-25 \mu \mathrm{m}$ region, which indicates that it is a favorable windscreen for both solar heating and radiative cooling. In this study, we chose low-density $20 \mu \mathrm{m}$ thick polyethylene film as the windscreen of collector A. Spectral transmittance of both windscreens in the $0.2-25 \mu \mathrm{m}$ region was tested using the UV-Vis-NIR spectrophotometer and FTIR spectrophotometer. The test results are shown in Fig. 8. 


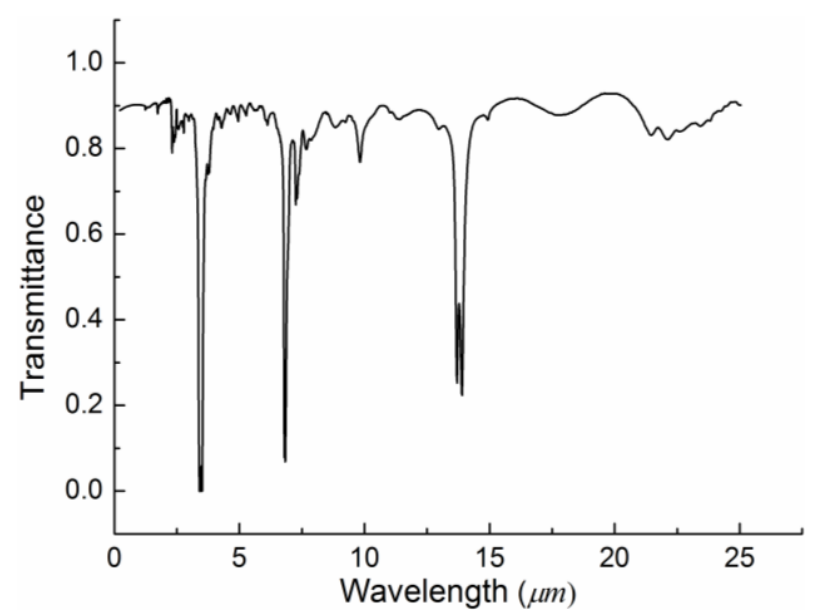

(a)

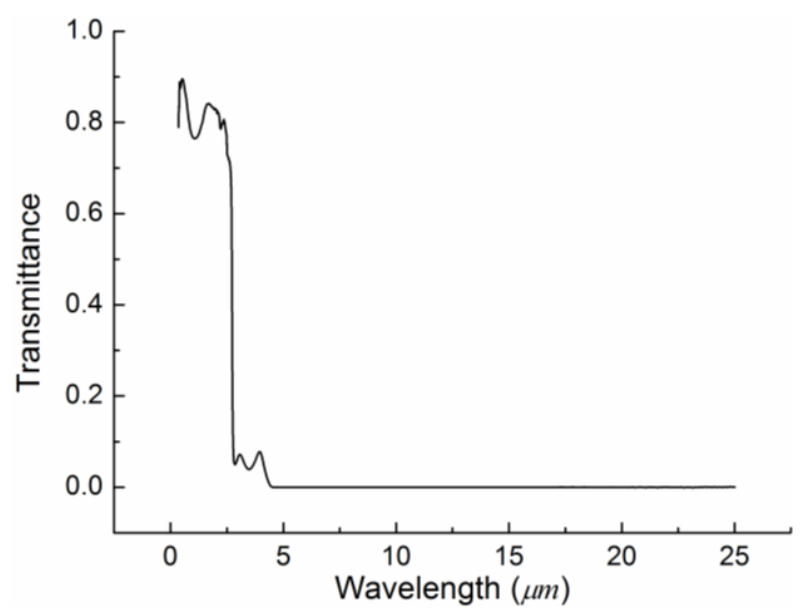

(b)

Fig. 8. Spectral transmittance of (a) $20 \mu \mathrm{m}$ thick low-density polyethylene film and (b) $5 \mathrm{~mm}$ thick glass plate

\subsection{Experimental set-up}

The experimental set-ups of systems A and B are shown in Fig. 9, which mainly consists of collector A, collector B, a thermostatic water tank, two circulation pumps, two water flow meters, and four platinum resistances. To carry out the daily average thermal performances of the two systems, in diurnal system testing mode the thermostatic water tank was replaced by two $65 \mathrm{~L}$ water tanks, and the circulation loops were changed to those of other common solar water heating systems ${ }^{[16]}$.

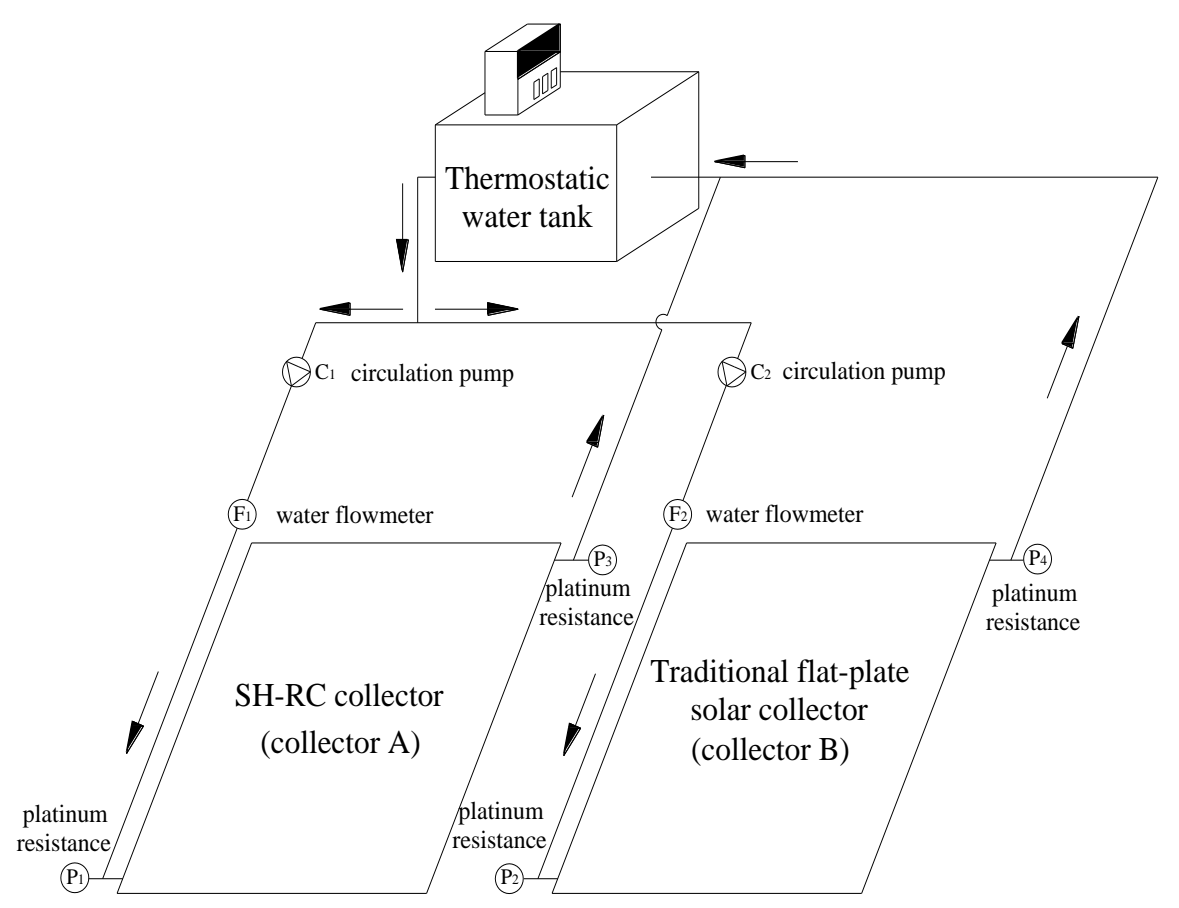

(a) 


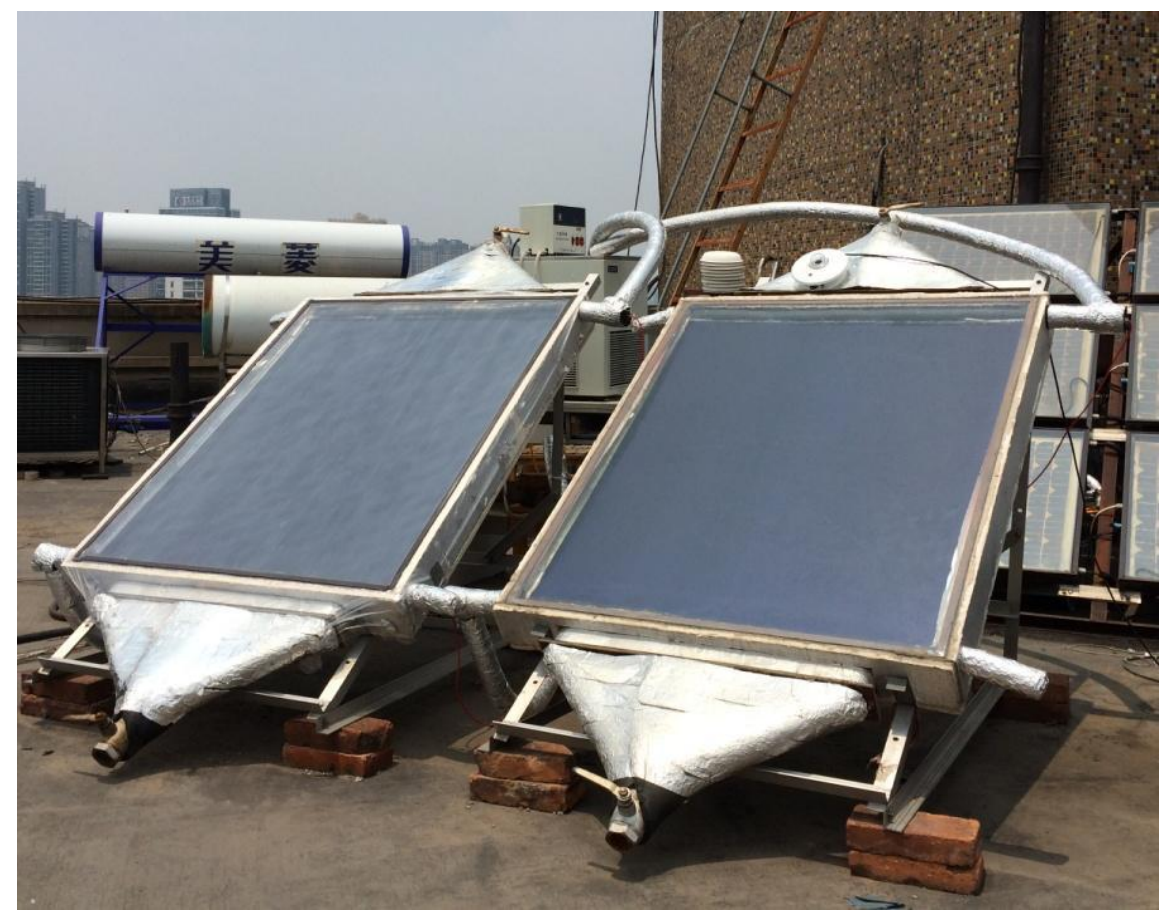

(b)

Fig. 9. Configuration of the experimental set-ups of systems A and B (a) schematic and (b) photo.

The experimental facilities were mounted on the roof platform of a building in the University of Science and Technology of China, Hefei $\left(32^{\circ} \mathrm{N}, 117^{\circ}\right.$ E). The two collectors were fixed at an inclination angle of $32^{\circ}$ facing south to maximize the annual total solar irradiance received, although collector A would have the best radiative cooling performance if it were installed horizontally with an unobstructed view of the sky ${ }^{[34]}$.

A list of experimental testing and monitoring devices is provided in Table 1. Solar irradiance was Table 1 List of experimental testing and monitoring devices

\begin{tabular}{ccc}
\hline Device & Specification & Accuracy \\
\hline Pyranometer & TBQ-2A & $2 \%$ \\
Water flow meter & Senlod LZB-10 & $2.5 \%$ \\
Platinum resistances & WZP-PT100 & $\pm 0.15^{\circ} \mathrm{C}$ \\
Thermocouple & Type T (copper-constantan) & $\pm 0.5{ }^{\circ} \mathrm{C}$ \\
Data acquisition/switch unit & Agilent 3490A & $/$ \\
\hline
\end{tabular}


measured using a TBQ-2A Pyranometer. The flow rate of circulating water was measured by two Senlod LZB-10 water flow meters, and four platinum resistances were installed at the inlets and outlets of each collector separately. The ambient temperature was measured by three thermocouples located in a thermometer shelter. All data were recorded using an Agilent data acquisition instrument with an interval of $10 \mathrm{~s}$.

\section{Performance evaluation of the systems and experimental methods}

\subsection{Performance evaluation of the system}

The diurnal thermal efficiency of the two collectors can be defined as the heat gain of the working fluid between the outlet and inlet of the collector divided by incident solar irradiance and aperture area, expressed as

$$
\eta_{\text {th }}=\frac{\dot{m} c_{w}\left(T_{o u t}-T_{\text {in }}\right)}{G A_{c}}
$$

where is $\dot{m}$ the mass flow rate of circulation water $(\mathrm{kg} / \mathrm{s}), c_{w}$ is the specific heat capacity of water $(\mathrm{J} /(\mathrm{kg} \cdot \mathrm{K})), T_{\text {in }}$ and $T_{\text {out }}$ are respectively water temperatures at the inlet and outlet of the collector $(\mathrm{K}), G$ is the incident solar irradiance per square meter $\left(\mathrm{W} / \mathrm{m}^{2}\right)$, and $A_{c}$ is the aperture area of the collector $\left(\mathrm{m}^{2}\right)$.

To eliminate the effect of solar irradiance variation and temperature difference between the surroundings and water at the collector inlet and to obtain general conclusions, the Hottel-Whillier model was applied. The heat gain of the collector can be expressed as ${ }^{[35]}$

$$
Q_{u}=A_{c}\left[G F_{R}(\tau \alpha)_{e}-F_{R} U_{L}\left(T_{i n}-T_{a}\right)\right]
$$

where $F_{R}$ is the heat removal factor of the collector, $(\tau \alpha)_{e}$ is the effective transmittance-absorptance product, $U_{L}$ is the energy loss coefficient of collector $\left(\mathrm{K} \cdot \mathrm{m}^{2} / \mathrm{W}\right)$, and $T_{a}$ is the ambient temperature $(\mathrm{K})$. 
Thus, the diurnal thermal efficiencies of the collector can be plotted as a function of reduced temperature, expressed as ${ }^{[36]}$

$$
\eta_{t h}=F_{R}(\tau \alpha)_{e}-F_{R} U_{L} \frac{T_{i n}-T_{a}}{G}
$$

where the intercept $F_{R}(\tau \alpha)_{e}$ and slope $F_{R} U_{L}$ represent the thermal efficiency of collector at zero-reduced temperature and the energy loss of the collector, respectively.

The daily average thermal efficiency of the system depends on the total solar irradiance of the aperture area, initial and final water temperature in the water tank, and mass of water in the water tank. This factor can be written as

$$
\overline{\eta_{t h}}=\frac{Q_{w}}{H}=\frac{m c_{w}\left(T_{\text {final }}-T_{\text {initial }}\right)}{H}
$$

where $Q_{w}$ is the total heat gain of water in the water tank during the testing time (J), $H$ is the total solar irradiance of the aperture area during the testing time $(\mathrm{J}), m$ is the mass of water in water tank $(\mathrm{kg})$, and $T_{\text {initial }}$ and $T_{\text {final }}$ are the initial and final water temperatures in the water tank $(\mathrm{K})$, respectively. In this study, water temperature in the water tank is the measured averages of five thermocouples in the water tank:

$$
T_{w}=\frac{\sum_{1}^{5} T_{i}}{5}
$$

where $T_{i}$ is the measured value of water temperature in the water tank by thermocouples $(\mathrm{K})$.

With different initial water temperatures in the water tank, the daily average thermal efficiency of the system can be linearly fitted as a function of reduced temperature, expressed as ${ }^{[37]}$

$$
\overline{\eta_{t h}}=\overline{\eta_{0}}-U_{\text {loss }} \frac{T_{\text {initial }}-\overline{T_{a}}}{H}
$$


The intercept $\overline{\eta_{0}}$ is the thermal efficiency of the system at zero-reduced temperature, slope $U_{\text {loss }}$ is the energy loss coefficient of the system $\left(\mathrm{K} \cdot \mathrm{m}^{2} / \mathrm{W}\right)$, and $\overline{T_{a}}$ represents the average ambient temperature during the testing time $(\mathrm{K})$.

The nocturnal cooling power of the two collectors can be defined as the heat loss of the working fluid between the outlet and inlet of the collector divided by the aperture area of the collector, expressed as

$$
P=\frac{\dot{m} c_{w}\left(T_{\text {in }}-T_{\text {out }}\right)}{A_{c}}
$$

To eliminate the effect of variation of temperature difference between the ambient and the water at the collector inlet and to obtain general conclusions, we proposed a similar model to express the cooling power of the collector by referencing the Hottel-Whillier model:

$$
P=A_{c}\left[F_{R}(\tau \alpha)_{e}+F_{R} U_{L}\left(T_{i n}-T_{a}\right)\right]
$$

The intercept $A_{c} F_{R}(\tau \alpha)_{e}$ and slope $A_{c} F_{R} U_{L}$ represent the cooling power of the collector at zero-reduced temperature and energy loss of the collector, respectively.

\subsection{Experimental methods}

In collector testing modes, as shown in Fig. 9, the two collectors were installed in parallel. The outlet of the thermostatic water tank was connected with the inlets of the two collectors by aluminium plastic tubes, which enables the thermostatic water tank to vary and unify the inlet water temperature of the two collectors. The inlet of the thermostatic water tank was connected with the outlets of the two collectors by aluminium plastic tubes as well. Four platinum resistances were installed at the inlet and outlet of each collector separately to monitor the inlet and outlet temperatures of the collector. Two water flow meters, one for each circulation loop, were set between the outlet of the thermostatic water tank and the inlet of the collector to record the circulation flow rate. In this study, the circulation flow 
rates of the two circulation loops were equated, about $0.016 \mathrm{~kg} / \mathrm{s}$ for the diurnal collector testing mode and about $0.012 \mathrm{~kg} / \mathrm{s}$ for the nocturnal collector testing mode. By changing the inlet water temperature of the collector under different weather conditions, a series of experiments was carried out under quasi-steady-state conditions to evaluate the thermal performance of the two collectors both at daytime and nighttime.

In the diurnal system testing mode, the thermostatic water tank was replaced by two $65 \mathrm{~L}$ water tanks and the circulation loops were changed to those of other common solar water heating systems. Thus, the two systems operated separately. In this study, long-term tests were carried out in good weather conditions from 8:00 a.m. to 4:00 p.m. during April to May in 2015. The initial water temperature in the water tank of the two systems was almost equated before each experiment and the circulation flow rates of the two systems were about $0.016 \mathrm{~kg} / \mathrm{s}$.

\section{Experimental results and discussions}

\subsection{Diurnal collector tests}

The diurnal collector tests were conducted on July 13, 2015. By changing the inlet temperatures, a series of diurnal thermal efficiency data of the two collectors was obtained. The curves of $\eta_{t h}$ against $\left(T_{i n}-T_{a}\right) / G$ are shown in Figs. 10(a) and 10(b), respectively. According to Equation (4), the thermal efficiencies of the two collectors can be expressed with regression lines:

$$
\begin{aligned}
& \eta_{t h-A}=0.627-8.44 \frac{T_{i n}-T_{a}}{G} \\
& \eta_{t h-B}=0.726-4.86 \frac{T_{i n}-T_{a}}{G}
\end{aligned}
$$

We learned that the thermal efficiency of collector A at zero-reduced temperature was $62.7 \%$, which was about $86.4 \%$ of that of collector B. As the $\left(T_{i n}-T_{a}\right) / G$ value increased, the thermal efficiency of both the two collectors decreased gradually with slopes of -8.44 and -4.86 , respectively, which 
indicates that the decline velocity of thermal efficiency of collector A is greater than that of collector B. Overall, the thermal efficiencies of collector A were lower than that of collector B under same $\left(T_{i n}-T_{a}\right) / G$ values based on the fact that the windscreen of the two collectors, namely, polyethylene film and glass plate, showed completely opposite transmittance properties in infrared bands. Moreover, the TPET composite surface of collector A has greater emissivities and thus greater radiative heat loss than the Ti-based solar absorber of collector B in infrared wavelengths, especially in the atmospheric window band. However, collector A still showed a significant solar heating performance.

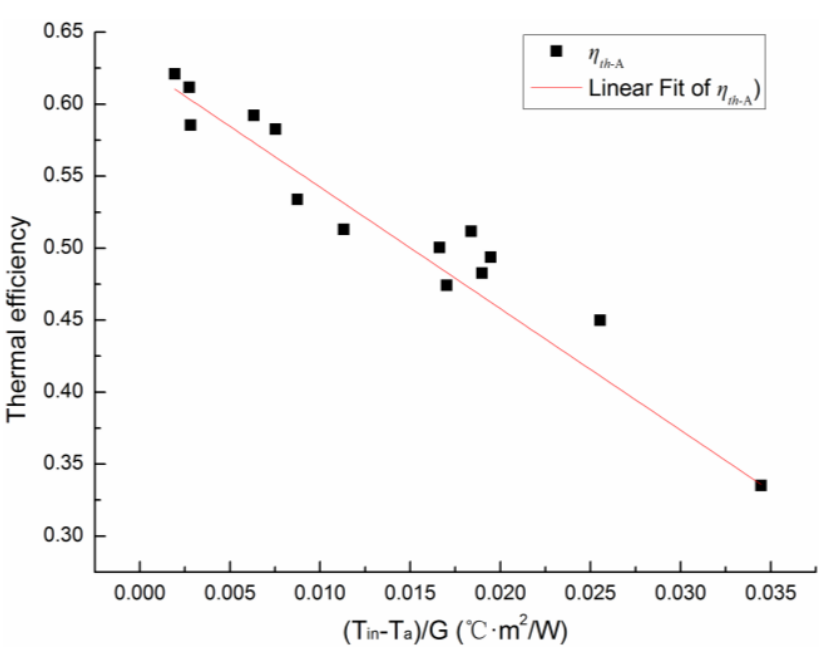

(a)

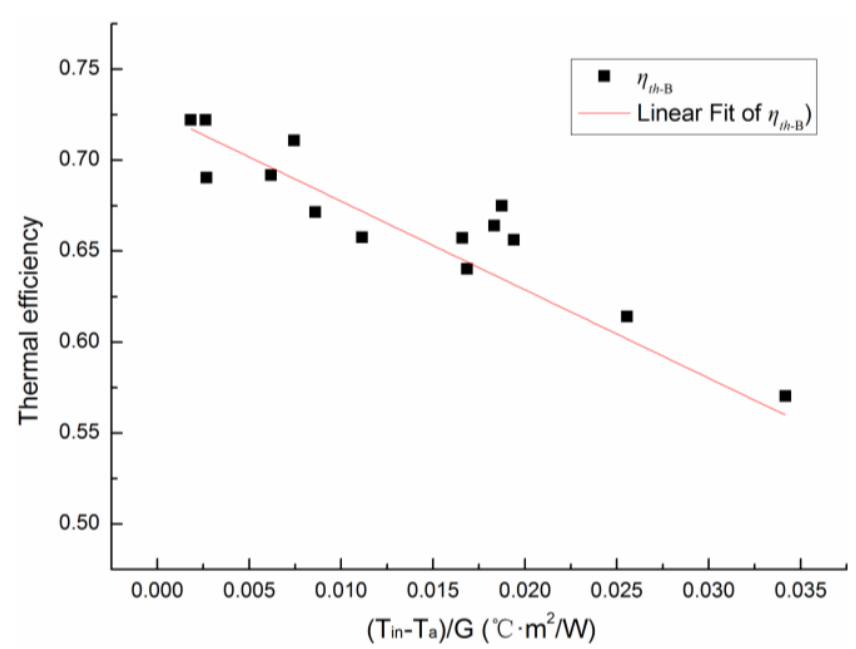

(b)

Fig. 10 Plot of diurnal thermal efficiencies of the two collectors:

(a) the SH-RC collector (collector A) and (b) the traditional flat-plate solar heating collector (collector B).

\subsection{Nocturnal collector tests on a clear night}

The nocturnal collector tests were first carried out in the late evening of July 14, 2015 to test the nocturnal cooling performance on a clear night. The curves of $P$ against $T_{i n}-T_{a}$ are shown in Figs. 11(a) and 11(b), respectively. According to Equation (8), the cooling powers of the two collectors can be expressed with regression lines:

$$
\begin{aligned}
& P_{A}=50.3+6.96\left(T_{i n}-T_{a}\right) \\
& P_{B}=6.12+3.93\left(T_{i n}-T_{a}\right)
\end{aligned}
$$


As seen from the figures, the nocturnal cooling power of collector A at zero-reduced temperature reached over $50 \mathrm{~W} / \mathrm{m}^{2}$, which represents the net radiative cooling power that is about eight times that of collector B. As the $T_{i n}-T_{a}$ value increased, the cooling power of both collectors increased gradually with slopes of 6.96 and 3.93, demonstrating that the increasing velocity of thermal efficiency of collector A is greater than that of collector B. In addition, when the water at the inlet of collector A reached its stagnation temperature where the cooling power fell to zero, the temperature was about $7.2{ }^{\circ} \mathrm{C}$ lower than that of the surroundings. By contrast, the stagnation temperature of the water at the inlet of collector B was just about $1.6{ }^{\circ} \mathrm{C}$ lower than the ambient temperature. Notably, the significant difference between the spectral properties of the windscreen and collecting surface in the infrared wavelengths of the two collectors resulted in the significantly different nocturnal radiative cooling performances. Furthermore, collector A in this study was fixed at an inclination angle of $32^{\circ}$ facing south to maximize the annual total solar irradiance received. However, greater net radiative cooling powers and lower stagnation temperatures of the water at the inlet of the collector would be expected if the collector was installed at any inclination angles less than $32^{\circ}$ or was mounted horizontally with an unobstructed view of the sky.

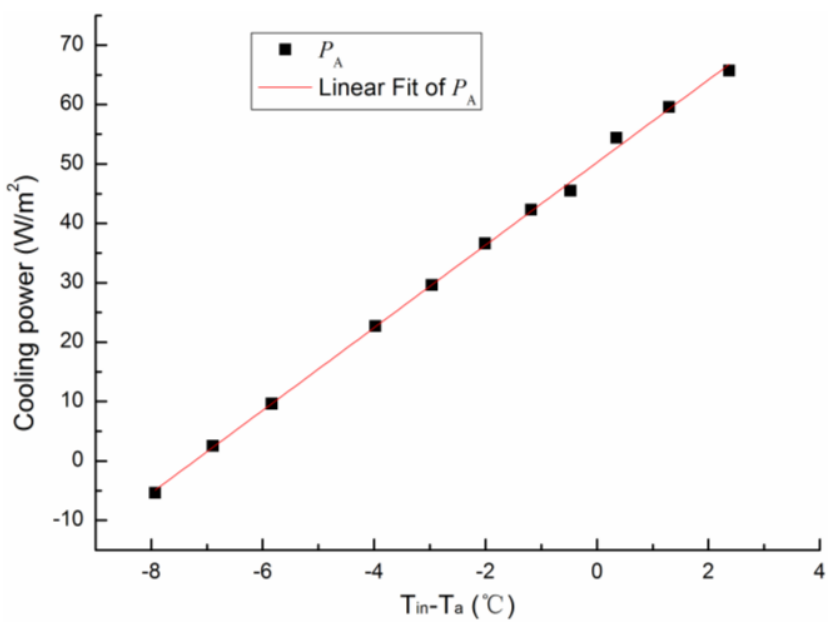

(a)

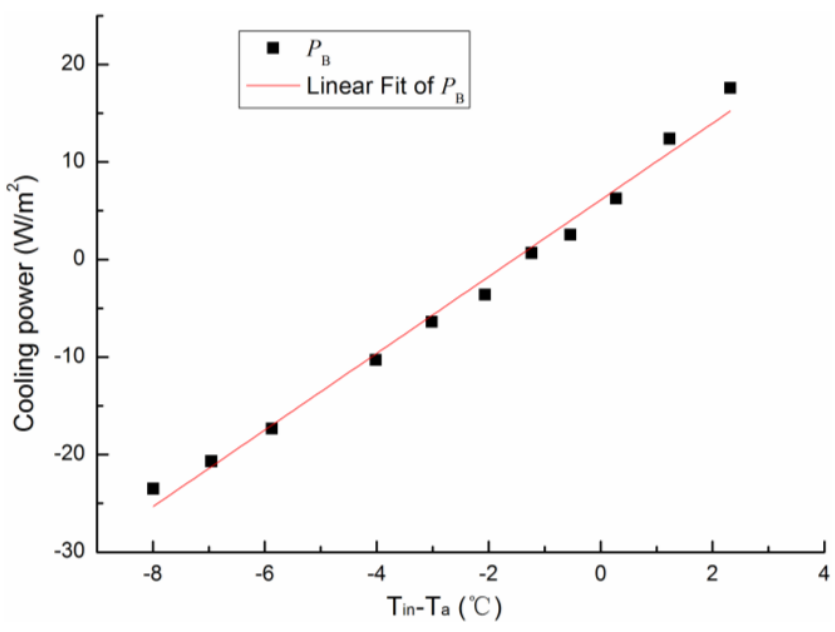

(b)

Fig. 11 Plot of nocturnal cooling power of the two collectors on a clear night: (a) the SH-RC collector (collector A) and (b) the traditional flat-plate solar heating collector (collector B). 


\subsection{Nocturnal collector tests on an overcast night}

The nocturnal collector tests were then carried out in the late evening of July 17, 2015 to test the nocturnal cooling performance during an overcast night. The curves of $P$ against $T_{i n}-T_{a}$ are shown in Figs. 12(a) and 12(b), respectively. According to Equation (9), the cooling powers of the two collectors can be expressed with regression lines:

$$
\begin{aligned}
& P_{A}=23.4+6.18\left(T_{i n}-T_{a}\right) \\
& P_{B}=2.76+3.91\left(T_{i n}-T_{a}\right)
\end{aligned}
$$

Fig. 12 reveals similar trends of cooling power variation, as shown in Fig. 11. The net radiative cooling power of the two collectors were 23.4 and $2.76 \mathrm{~W} / \mathrm{m}^{2}$, which amounted to about half of that on a clear night. However, the increasing velocities (i.e., slopes) of the cooling power of both collectors were almost the same as that on a clear night. Additionally, the stagnation temperatures of the water at the inlet of the two collectors were about 3.8 and $0.71{ }^{\circ} \mathrm{C}$. Comparing the values of the net radiative cooling power and stagnation temperature between a clear night and an overcast night, we learned that the sky condition is a significant factor in the radiative cooling performance of the collector. The clearer the sky, the better radiative cooling performance the collector will exhibit.

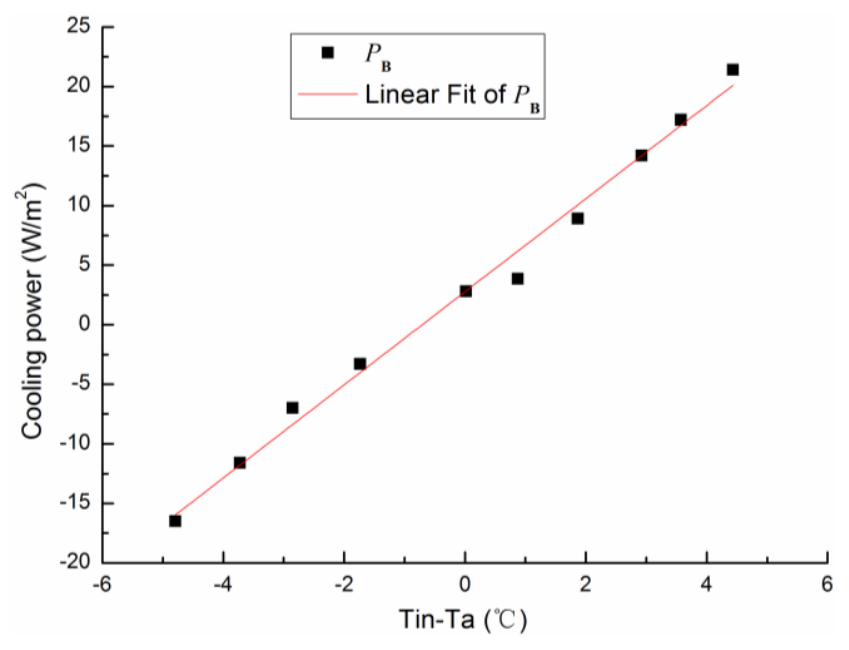

(a)

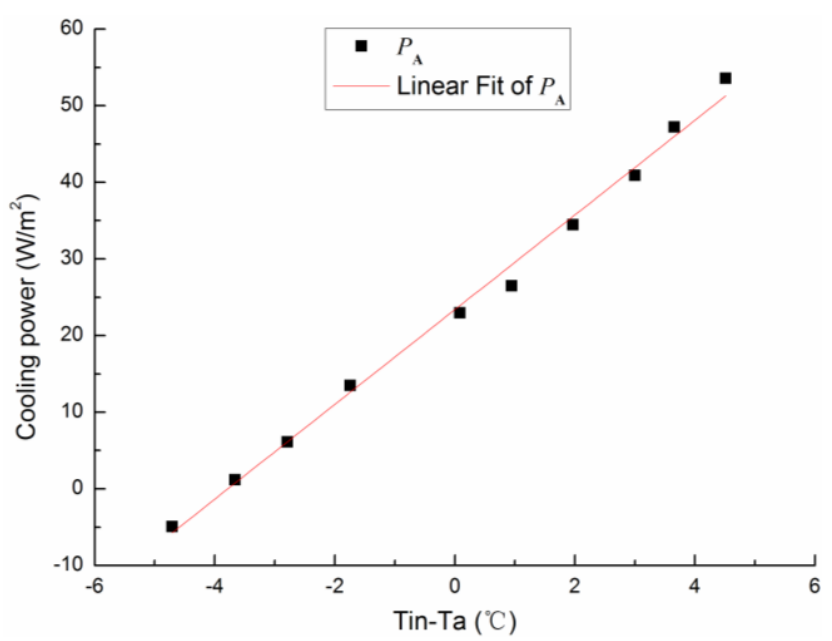

(b)

Fig. 12 Plot of nocturnal cooling power of the two collectors on an overcast night: (a) the SH-RC collector (collector A) and (b) the traditional flat-plate solar heating collector (collector B). 
Table 2 shows the thermal performance of different types of flat plate solar water collector, radiative cooling collector and the $\mathrm{SH}-\mathrm{RC}$ collector. The thermal efficiencies or cooling powers at zero reduced temperature are chosen to be compared. We can see from Table 2 that the SH-RC collector shows satisfactory performances both at daytime and nighttime. The new design makes it possible to operate efficiently throughout all day and has potential for various applications. Taking residential applications as an example, heat and cooling energy are produced for domestic use in different time periods and/or seasons.

Table 2 Thermal performance of different types of flat plate collector

\begin{tabular}{ccc} 
Type & Thermal efficiency & $\begin{array}{c}\text { Net cooling power (clear sky) } \\
\left(\mathrm{W} / \mathrm{m}^{2}\right)\end{array}$ \\
\hline Solar water collector $^{[38]}$ & $0.728 \sim 0.790$ & - \\
Radiative cooling collector $^{[39]}$ & - & $20 \sim 80$ \\
SH-RC collector & 0.627 & 50.3 \\
\hline
\end{tabular}

\subsection{Diurnal system tests}

In general, the system performance was of greater concern. In this study, a multiday system-wide solar heating test was performed from April to May 2015 to determine the daily solar heating capacity of the collector. The multiday results of systems A and B are shown in Fig. 13. According to the linear fitted curves, the daily average thermal efficiencies are expressed as

$$
\begin{aligned}
& \overline{\eta_{t h-A}}=0.386-0.187 \frac{T_{\text {initial }}-\overline{T_{a}}}{H} \\
& \overline{\eta_{t h-B}}=0.484-0.136 \frac{T_{\text {initial }}-\overline{T_{a}}}{H}
\end{aligned}
$$

The thermal efficiency of system A at zero-reduced temperature was $38.6 \%$, which is about $79.8 \%$ of that of system B. As the $\left(T_{\text {initial }}-\overline{T_{a}}\right) / H$ value increased, the thermal efficiency of both the two 
systems decreased steadily with slopes of -0.187 and -0.136 . Although system A was lower than system B in daily average thermal efficiency, the former still presented a considerable solar heating capacity.

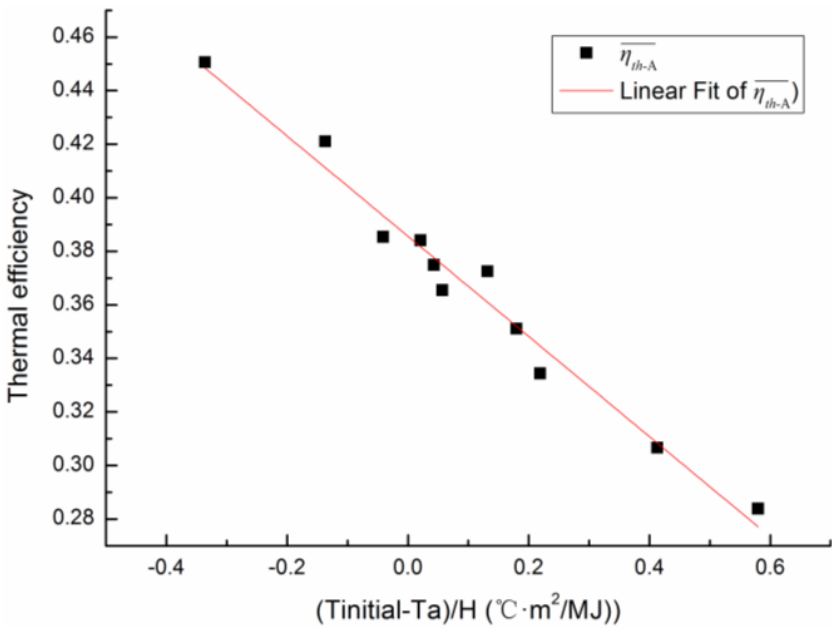

(a)

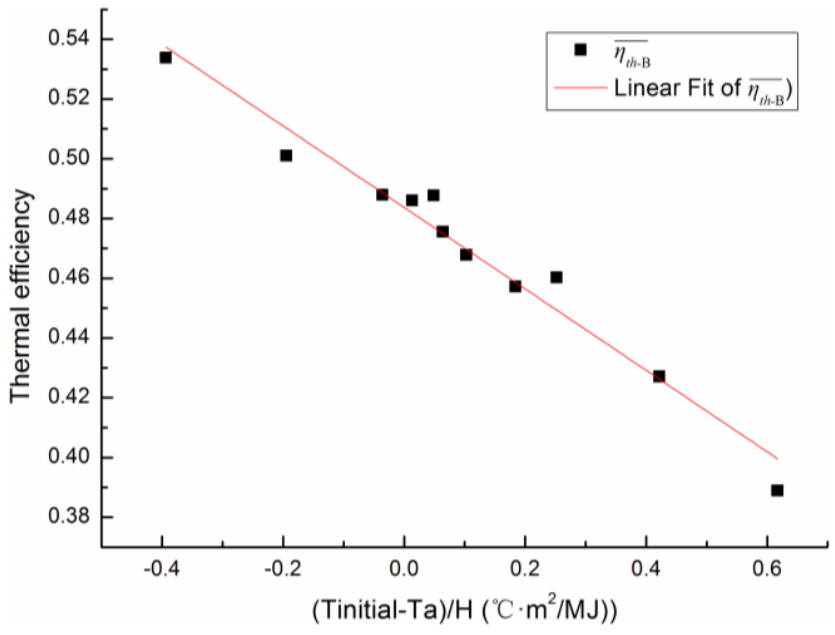

(b)

Fig. 13 Plot of daily average thermal efficiencies of the two systems:

(a) the SH-RC system (system A) and (b) the traditional flat-plate solar heating system (system B).

\section{Conclusions}

In this paper, a type of composite surface that combined Ti-based solar selective absorber coating with TPET was trial-manufactured to achieve integrative functions of both solar heating and radiative cooling. The spectral properties of the TPET composite surface were measured with spectral testing instruments. Additionally, a combined system based on the TPET composite surface (named SH-RC system) was mounted together with a traditional flat-plate solar heating system. Comparative experiments were carried out to investigate their thermal performances both at daytime and nighttime. The results are as follows:

(1) The TPET composite surface had high spectral absorptivity (emissivity) in solar radiation and atmospheric window wavelengths, indicating that it could act as a solar collector during the day and a nocturnal radiator during the night. In other bands aside from the solar radiation and atmospheric window bands, the TPET composite surface exhibited relatively low spectral absorptivity (emissivity), 
allowing it to decrease radiative heating loss during daytime and radiative cooling loss during nighttime.

(2) In diurnal collector testing mode, thermal efficiency of the SH-RC collector at zero-reduced temperature was $62.7 \%$, which was about $86.4 \%$ of that of the traditional flat-plate solar heating collector, showing a significant solar heating performance.

(3) In nocturnal collector testing mode, the SH-RC collector had net radiative cooling powers of $50.3 \mathrm{~W} / \mathrm{m}^{2}$ on a clear night and $23.4 \mathrm{~W} / \mathrm{m}^{2}$ on an overcast night, presenting a considerable radiative cooling capacity. The stagnation temperatures of water at the inlet of the two collectors were 7.2 and $3.8{ }^{\circ} \mathrm{C}$ in the two sky conditions. Additionally, better performance would be expected if the collector was installed at any inclination angles less than $32^{\circ}$. By contrast, the traditional flat-plate solar heating collector exhibited very low radiative cooling capacity.

(4) In diurnal system testing mode, the daily average thermal efficiency of the SH-RC system and the traditional flat-plate solar heating system at zero-reduced temperature was $38.6 \%$ and $48.4 \%$, respectively.

(5) Overall, the SH-RC system showed a considerable performance for both diurnal solar heating and nocturnal radiative cooling. The system could work all day to address the limitations of traditional radiative cooling systems and flat-plate solar systems and to shorten the initial payback period.

The spectral property of the collecting surface, the windscreen material, the sky condition, the humidity of the testing environment, and the thermal insulation are the main factors in determining the performance of the SH-RC system. These parameters could be further studied to explore thermal behavior, especially the limited nocturnal radiative cooling capacity of the SH-RC system.

\section{Acknowledgements}


The study was sponsored by (1) the National Science Foundation of China (NSFC 51476159, 51178442, 51206154 and 51408578), (2) the Fundamental Research Funds for the Central Universities, and (3) Dongguan Innovative Research Team Program (No. 2014607101008).

\section{References}

[1] Luis Pérez-Lombard, José Ortiz, Christine Pout. A review on buildings energy consumption information. Energy and Building, 2008; 40:394-398.

[2] Y. Tian, C.Y. Zhao. A review of solar collectors and thermal energy storage in solar thermal applications. Applied Energy, 2013; 104:538-553.

[3] Musa T. Zarmai, N.N. Ekere, C.F. Oduoza, et al. A review of interconnection technologies for improved crystalline silicon solar cell photovoltaic module assembly. Applied Energy, 2015; $154: 173-182$.

[4] D. Rodríguez-Sánchez, J.F. Belmonte, M.A. Izquierdo-Barrientos, et al. Solar energy captured by a curved collector designed for architectural integration. Applied Energy, 2014; 116:66-75.

[5] S. Jahangiri Mamouri, H. Gholami Derami, M. Ghiasi, et al. Experimental investigation of the effect of using thermosyphon heat pipes and vacuum glass on the performance of solar still. Energy, 2014; 75:501-507.

[6] Z. Abdin, M.A. Alim, R. Saidur, et al. Solar energy harvesting with the application of nanotechnology. Renewable and Sustainable Energy Reviews, 2013; 26:837-852.

[7] Xiao Jin, Weifu Sun, Zihan Chen, et al. Development and performance of a dual tank solar-assisted heat pump system. Applied Energy, 2015; 149:125-132. 
[8] Yuehong Su, Saffa B. Riffat, Gang Pei. Comparative study on annual solar energy collection of a novel lens-walled compound parabolic concentrator (lens-walled CPC). Sustainable Cities and Society, 2012; 4:35-40.

[9] Xiande Fang, Dingkun Li. Solar photovoltaic and thermal technology and applications in China. Renewable and Sustainable Energy Reviews, 2013; 23:330-340.

[10] Aldo Orioli, Alessandra Di Gangi. Review of the energy and economic parameters involved in the effectiveness of grid-connected PV systems installed in multi-storey buildings. Applied Energy, 2014; 113:955-969.

[11] Guiqiang Li, Gang Pei, Jie Ji, et al. Outdoor overall performance of a novel air-gap-lens-walled compound parabolic concentrator (ALCPC) incorporated with photovoltaic/thermal system. Applied Energy, 2015; 144:214-223.

[12] Jie Ji, Yanqiu Wang, Weiqi Yuan, et al. Experimental comparison of two PV direct-coupled solar water heating systems with the traditional system. Applied Energy, 2014; 136:110-118.

[13] Pei Gang, Fu Huide, Zhang Tao, et al. A numerical and experimental study on a heat pipe PV/T system. Solar Energy, 2012; 85:911-921.

[14] M. Zukowski. Experimental investigations of thermal and flow characteristics of a novel microjet air solar heater. Applied Energy, 2015; 142:10-20.

[15] Shane Colclougha, Teresa McGrath. Net energy analysis of a solar combi system with Seasonal Thermal Energy Store. Applied Energy, 2015; 147:611-616.

[16] E. Halawa, K.C. Chang, M. Yoshinaga. Thermal performance evaluation of solar water heating systems in Australia, Taiwan and Japan - A comparative review. Renewable Energy, 2015; 83:1279-1286. 
[17] Y.H. Yau, S. Hasbi. A review of climate change impacts on commercial buildings and their technical services in the tropics. Renewable and Sustainable Energy Reviews, 2013; 18:430-441.

[18] Zhi Yu, Jie Ji, Wei Sun, et al. Experiment and prediction of hybrid solar air heating system applied on a solar demonstration building. Energy and Buildings, 2014; 78:59-65.

[19] Gilles Notton, Fabrice Motte, Christian Cristofari, et al. Performances and numerical optimization of a novel thermal solar collector for residential building. Renewable and Sustainable Energy Reviews, 2014; 33:60-73.

[20] Elias M. Lushiku, Claes-Goran Granqvist. Radiative cooling with selectively infrared-emitting gases. Applied Optics, 1984; 23 (11):1835-1843.

[21] A.R. Gentle, K.L. Dybdal, G.B. Smith. Polymeric mesh for durable infra-red transparent convection shields: Applications in cool roofs and sky cooling. Solar Energy Materials and Solar Cells, 2013; 115:79-85.

[22] Moien Farmahini Farahani, Ghassem Heidarinejada, Shahram Delfani. A two-stage system of nocturnal radiative and indirect evaporative cooling for conditions in Tehran. Energy and Buildings, 2010; 42:2131-2138.

[23] Aaswath P. Raman, Marc Abou Anoma, Linxiao Zhu, et al. Passive radiative cooling below ambient air temperature under direct sunlight. Nature, 2014; 515:540-544.

[24] Eden Rephaeli, Aaswath P. Raman, Shanhui Fan. Ultrabroadband photonic structures to achieve high-performance daytime radiative cooling. Nano Letter, 2013; 13:1457-1461.

[25] A. R. Gentle, and G. B. Smith. Radiative heat pumping from the earth using surface phonon resonant nanoparticles. Nano Letter, 2010; 10:373-379.

[26] A. R. Gentle, J. L. C. Aguilar, G. B. Smith. Optimized cool roofs: Integrating albedo and thermal emittance with R-value. Solar Energy Materials and Solar Cells, 2011; 95:3207-3215. 
[27] S.N. Bathgate, S.G. Bosi. A robust convection cover material for selective radiative cooling applications, Solar Energy Materials and Solar Cells, 2011; 95:2778-2785.

[28] Yi Man, Hongxing Yang, Jeffrey D. Spitler, et al. Feasibility study on novel hybrid ground coupled heat pump system with nocturnal cooling radiator for cooling load dominated buildings. Applied Energy, 2011; 88:4160-4171.

[29] Eden Rephaeli, Aaswath Raman, Shanhui Fan. Ultrabroadband Photonic Structures To Achieve High-Performance Daytime Radiative Cooling. Nano letter, 2013; 13:1457-1461.

[30] Joze Rakovec, Gregor Skok, Rahela Zabkar, et al. The influence of the depth of a very shallow cool-pool lake on nocturnal cooling. Agricultural and Forest Meteorology, 2015; 203:17-29.

[31] Mingke Hu, Gang Pei, Lei Li, et al. Theoretical and Experimental Study of Spectral Selectivity Surface for Both Solar Heating and Radiative Cooling. International Journal of Photoenergy, 2015 (2015) Article ID 807875, 9 pages.

[32] G. Mihalakakou, A. Ferrante, J.O. Lewis. The cooling potential of a metallic nocturnal radiator. Energy and Buildings, 1998; 28:251-256.

[33] M. Matsuta, S. Terada, H. Iro. Solar Heating And Radiative Cooling Using a Solar Collector-Sky Radiator With a Spectrally Selective Surface. Solar Energy, 1987; 39(3):183-186.

[34] Ewatar Erell, Yair Etzion. Heating experiments with a radiative cooling system. Building and Environment, 1996; 31(6):509-517.

[35] J.A. Duffie, W.A. Beckman. Solar engineering of thermal processes. 3rd ed. New York: Wiley-Interscience; 2006.

[36] ANSI/ASHRAE 93 - Methods of testing to determine the thermal performance of solar collectors. New York: ASHRAE Inc.; 2010. 
[37] Jie Ji, Jian ping Lu, T.T. Chow, et al. A sensitivity study of a hybrid photovoltaic/thermal water-heating system with natural circulation. Applied Energy, 2007; 84:222-237.

[38] D.E. Roberts. A figure of merit for selective absorbers in flat plate solar water heaters. Solar Energy, 2013; 98:503-510.

[39] Ursula Eicker, Antoine Dalibard. Photovoltaic-thermal collectors for night radiative cooling of buildings. Solar Energy, 2011; 85:1322-1335. 